\title{
Spin-Label ESR of Bacteriophage M13 Coat Protein in Mixed Lipid Bilayers. Characterization of Molecular Selectivity of Charged Phospholipids for the Bacteriophage M13 Coat Protein in Lipid Bilayers ${ }^{\dagger}$
}

\author{
Cor J. A. M. Wolfs, ${ }^{*, \downarrow}$ Läszlo I. Horvăth, ${ }^{\S, \|}$ Derek Marsh, ${ }^{\S}$ Anthony Watts, ${ }^{\perp}$ and Marcus A. Hemminga ${ }^{\ddagger}$ \\ Department of Molecular Physics, Agricultural University, Dreijenlaan 3, $6703 \mathrm{HA}$ Wageningen, The Netherlands, \\ Max-Planck-Institut für biophysikalische Chemie, Abteilung Spektroskopie, D-3400 Göttingen, Federal Republic of Germany, \\ and Department of Biochemistry, University of Oxford, Oxford, United Kingdom \\ Received May 5, 1989; Revised Manuscript Received August 9, 1989
}

\begin{abstract}
Bacteriophage M13 major coat protein has been incorporated at different lipid/protein ratios in lipid bilayers consisting of various ratios of dimyristoylphosphatidylcholine (DMPC) to dimyristoylphosphatidylglycerol (DMPG). Spin-label ESR experiments were performed with phospholipids labeled at the C-14 position of the $s n-2$ chain. For M13 coat protein recombinants with DMPC alone, the relative association constants were determined for the phosphatidylcholine, phosphatidylglycerol, and phosphatidic acid spin-labels and found to be 1.0, 1.0, and 2.1 relative to the background DMPC, respectively. The number of association sites for each phospholipid on the protein was found to be 4 per protein monomer. The intrinsic off-rates for lipid exchange at the intramembranous surface of the protein in DMPC alone at $30^{\circ} \mathrm{C}$ were found to be $5 \times 10^{6}, 6 \times 10^{6}$, and $2 \times 10^{6} \mathrm{~s}^{-1}$ for the phosphatidylcholine, phosphatidylglycerol, and phosphatidic acid spin-labels, respectively. Adding DMPG to the DMPC lipid system increased the exchange rates of the lipids on and off the protein. By gel filtration chromatography, it is found that protein aggregation is reduced after addition of DMPG to the lipid system. This is in agreement with measurements of tryptophan fluorescence, which show a decrease in quenching efficiency after introduction of DMPG in the lipid system. The results are interpreted in terms of a model relating the ESR data to the size of the protein-lipid aggregates.
\end{abstract}

$T_{\text {he }}$ he nonenveloped filamentous bacteriophage M13, which is closely related to bacteriophages fd and fl (Ray, 1977), enters the Escherichia coli cell, leaving its major coat protein (a product of gene 8) in the cytoplasmic membrane (Marvin \& Wachtel, 1975). During DNA replication and synthesis of new phage proteins, the coat protein is stored in the membrane as an integral membrane protein. Newly synthesized coat protein, which arrives at the membrane as a preprotein and is converted to the mature coat protein by the action of a leader peptidase (Kühn et al., 1986), is stored in the membrane and encapsulates the viral DNA molecule during the membrane-associated assembly of new virus particles. The parental coat protein, after it is incorporated in the membrane, is also used for the formation of new infectious M13 bacteriophages (Armstrong et al., 1983). The coat protein of M13, whose amino acid sequence is known (Van Wezenbeek et al., 1980; Beck et al., 1978), consists of three different regions: a basic carboxyl terminus, a central hydrophobic core of 19 amino acids, and an acidic amino terminus (Chamberlain et al., 1978; Van Wezenbeek et al., 1980).

Spin-label electron spin resonance (ESR) ${ }^{1}$ has been shown to be a particularly useful technique for studying the interactions of M13 coat protein in reconstituted lipid systems (Datema et al., 1987a). Three groups of phospholipids could

\footnotetext{
${ }^{\dagger}$ This research was supported by the Commission of the European Communities (Contracts ST2J-0088 and ST2J-0368-C).

* Address correspondence to this author.

'Agricultural University.

Max-Planck-Institut für biophysikalische Chemie.

"Permanent address: Institute of Biophysics, Biological Research Centre, Szeged, Hungary.

$\perp$ University of Oxford.
}

be distinguished showing different specificities for the M13 coat protein (group I, CL and PA; group II, PG, PS, and SA; and group III, PC and PE). These spin-label experiments were performed in a mixed-phospholipid system consisting of DMPC (80 wt \%) and DMPG (20 wt \%). After reconstitution, this lipid system resulted in a stable, nonaggregating, homogeneous, clear vesicle solution. Recently, we have been able to incorporate M13 coat protein also in pure DMPC lipids so that now the lipid-protein interaction can be studied as a function of increasing content of negatively charged phospholipid. This is especially interesting, since in earlier experiments (Datema et al., 1987a) the protein was shown to have an increased specificity for negatively charged phospholipids.

The present paper examines the effect of M13 coat protein on mixed systems with variable amounts of DMPG and DMPC. The number of lipid association sites is determined in pure DMPC, as well as the relative association constants, for one representative spin-labeled phospholipid from each of the above three groups of phospholipids of different specificity. Furthermore, the lipid exchange rates at the protein interface have been determined, as a function both of the $\mathrm{L} / \mathrm{P}$ ratio and of the DMPG content of the reconstituted systems.

\footnotetext{
${ }^{1}$ Abbreviations: DMPC, 1,2-dimyristoylglycero-3-phosphocholine; DMPG, 1,2-dimyristoylglycero-3-phosphoglycerol; 14-PCSL, -PGSL, and -PASL, 1-acyl-2-[14-(4,4-dimethyloxazolidine- $N$-oxyl)stearoyl]glycero-3-phosphocholine, -phosphoglycerol, and -phosphoric acid; ESR, electron spin resonance; $\mathrm{CL}$, cardiolipin; PA, phosphatidic acid; PG, phosphatidylglycerol; PS, phosphatidylserine; SA, stearic acid; PC, phosphatidylcholine; PE, phosphatidylethanolamine; L/P, lipid to protein molar ratio; EDTA, ethylenediaminetetraacetic acid; Tris, tris(hydroxymethyl)aminomethane.
} 
Fluorescence self-quenching and gel filtration experiments were performed to obtain information about the protein packing and aggregation size.

\section{Materials and Methods}

Chemicals. DMPC and DMPG were obtained from Sigma Chemical Co. and were shown to be pure on thin-layer chromatography. 14-PCSL, 14-PGSL, and 14-PASL spinlabels have been synthesized from egg yolk lysophosphatidylcholine and the corresponding spin-labeled stearic acids as described in Marsh and Watts (1982). All other chemicals used were from Merck B.V. (The Netherlands) and were of analytical grade. Dialysis membrane, Spectra-Por 4, was obtained from Hicol B.V. (The Netherlands).

Sample Preparation. Bacteriophage M13 was grown as described (Garssen et al., 1977) and isolated by means of poly(ethylene glycol) 6000 precipitation and $\mathrm{CsCl}$ ultracentrifugation as described in Spruijt et al. (1989). The coat protein was isolated from the phage according to Knippers and Hofmann-Berling (1966). For preparation of the ESR samples, the method developed by Hagen et al. (1978) was used, with some modifications. In a test tube, $225 \mu \mathrm{g}(1.5 \mathrm{~mol} \%$ of the final lipid concentration) of spin-labeled phospholipid was evaporated from ethanol to dryness with nitrogen gas flow. Out of three stock solutions, DMPC, DMPG, and M13 coat protein $(15 \mathrm{mg} / \mathrm{mL}$ in $5 \mathrm{mM}$ Tris- $\mathrm{HCl}, \mathrm{pH} 8.0$, containing 8.0 M urea, $20 \mathrm{mM}$ ammonium sulfate, $0.2 \mathrm{mM}$ EDTA, and $2 \mathrm{wt} \%$ cholate), determined volumes were pipetted to obtain samples with the desired lipid composition, $\mathrm{L} / \mathrm{P}$ ratio, and final concentration (10 mg of lipid $/ \mathrm{mL}$ ). After incubation of the samples at $55^{\circ} \mathrm{C}$ for $1 \mathrm{~h}$, dialysis was started against $5 \mathrm{mM}$ Tris- $\mathrm{HCl}, \mathrm{pH} 8.0,0.02 \mathrm{mM}$ EDTA, and $10 \%(\mathrm{v} / \mathrm{v})$ methanol. Buffer changes were after 12,24 , and $36 \mathrm{~h}$. During the last dialysis step, methanol was omitted. In the case of $\mathrm{L} / \mathrm{P}$ ratios up to 15 , dialysis was carried out at $4^{\circ} \mathrm{C}$, and after dialysis, the samples were concentrated by freeze-drying and resuspended in $0.15 \mathrm{~mL}$ of water. Homogeneous multilamellar bilayers were obtained by vortexing and heating several times through the phase transition temperature. Samples with higher lipid/protein ratios were dialyzed at room temperature and concentrated by high-speed centrifugation $(1 \mathrm{~h}, 80000 \mathrm{~g}, 14$ $\left.{ }^{\circ} \mathrm{C}\right)$. At this point, aliquots of the samples were taken to determine the sample homogeneity by sucrose gradient centrifugation $\left(4 \mathrm{~h}, 182000 \mathrm{~g}, 4^{\circ} \mathrm{C}\right)$. All samples prepared showed one single, sharp band, indicating that vesicles of uniform $\mathrm{L} / \mathrm{P}$ ratio had been formed. Lipid and protein concentrations were determined by phosphate and protein determination according to Bartlett (1959) and Peterson (1977) and used to determine the lipid to protein ratio.

ESR Spectroscopy. A Varian E-12, 9-GHz spectrometer with nitrogen gas flow temperature regulation was used to record the ESR spectra. Small sample capillaries were accommodated within 4-mm quartz ESR tubes, containing silicone oil for thermal stability. Temperatures were measured with a thermocouple placed just above the cavity within the quartz tube. A Digital Equipment Corp. LPS system and a dedicated PDP 11/10 computer with a VT11 display were used to digitize the spectra. ESR spectrometer settings were those given in Datema et al. (1987a).

Spectral Simulations. Spectral simulations were carried out with a two-site chemical exchange model for the motionally restricted and fluid spin-label components (Horvâth et al., 1988). A summation was applied, corresponding to an unoriented membrane, over all angular orientations of the membrane normal. The simulation program corresponds to model I as presented by Davoust and Devaux (1982). The motionally restricted component used during simulations corresponded to a motionally restricted difference spectrum obtained by intersubtractions of the experimental spectra. This singlecomponent spectrum had a similar line shape to that of 14 PCSL in sonicated DMPC vesicles recorded at $4{ }^{\circ} \mathrm{C}$, which also gave good results in the spectral subtraction procedure. The mobile spectrum used was either 14-PCSL or 14-PGSL in the mixed-lipid system DMPC/DMPG $80 / 20 \mathrm{w} / \mathrm{w} \%$, recorded at $30^{\circ} \mathrm{C}$, the same temperature used to record the $\mathrm{L} / \mathrm{P}$ samples. Both single-component line shapes were adjusted in the first-derivative representation because this gave maximum sensitivity to the line-width parameters, and considerable iteration was used to adjust these parameters; the $g$ - and $A$ tensor values could be established more readily. The sum of least squares of differences between experimental and simulated spectra had a minimum value when the line heights were rather sensitive to subtle changes in the line widths, and the integrated intensity of the simulated spectrum accounted for the intensity of the experimental spectra within $\pm 3 \%$. It should be noted that two-component simulations in the first-derivative representation were particularly sensitive to the line broadening due to chemical exchange. Other details of the simulation procedure can be found in Horvâth et al. (1988).

Fluorescence. Fluorescence samples were prepared in a similar way as for the ESR samples except for differences in the $L / P$ ratio and the total concentration of lipid and protein. The starting protein concentration was $0.1 \mathrm{mg} / \mathrm{mL}$ and, as mentioned above, was determined after dialysis. The samples were measured directly after dialysis, on a Perkin-Elmer LS-5 luminesence spectrophotometer. Excitation was at $280 \mathrm{~nm}$, and emission was recorded from 300 to $400 \mathrm{~nm}$. Emission and excitation slits were set at $5 \mathrm{~nm}$. Measurements were performed in a $3.0-\mathrm{mL}$ cuvette in a temperature-controlled cuvette housing. In order to compare different series of experiments, the spectra were scaled to $0.1 \mathrm{mg} / \mathrm{mL}$ protein concentration.

Gel Filtration. After fluorescence experiments, the samples were incubated for $1.5 \mathrm{~h}$ in $50 \mathrm{mM}$ cholate, $10 \mathrm{mM}$ Tris, and $0.2 \mathrm{mM}$ EDTA, pH 8.0, loaded on a Superose- 6 column (Pharmacia), and eluted with the same buffer at a flow rate of $0.25 \mathrm{~mL} / \mathrm{min}$. Detection was at $280 \mathrm{~nm}$ [see also Spruijt et al. (1989)].

\section{RESULTS}

The ESR spectra of 14-PCSL in M13 coat protein/DMPC complexes of different $\mathrm{L} / \mathrm{P}$ mole ratios are given in Figure 1. The spectra were recorded at $30^{\circ} \mathrm{C}$, which is well above the phase transition temperature of DMPC $\left(23^{\circ} \mathrm{C}\right)$. The spectra display two well-resolved components: a sharp, three-line component corresponding to lipids in the bulk of the bilayer, undergoing weakly anisotropic motion; and a second broad component, resolved in the outer wings of the spectrum, representing labels undergoing restricted motion, relative to the bulk phospholipids. This second component has previously been assigned to phospholipid acyl chains interacting directly with the protein in the membrane (Datema et al., 1987a). This is confirmed by the increase in the relative proportion of the broad component with increasing amount of protein incorporated in the bilayer.

The fraction of motionally restricted spin-labeled component, $f$, can be quantitated independently either by spectral subtraction [see, e.g., Marsh (1982); Marsh and Watts (1982), and Datema et al. (1987a)] or by two-component spectral simulation using the exchange-coupled Bloch equations as described for the myelin proteolipid protein and for rhodopsin (Horvāth et al., 1988; Ryba et al., 1987). The two methods are found to give similar results. $\mathrm{L} / \mathrm{P}$ titrations obtained in 


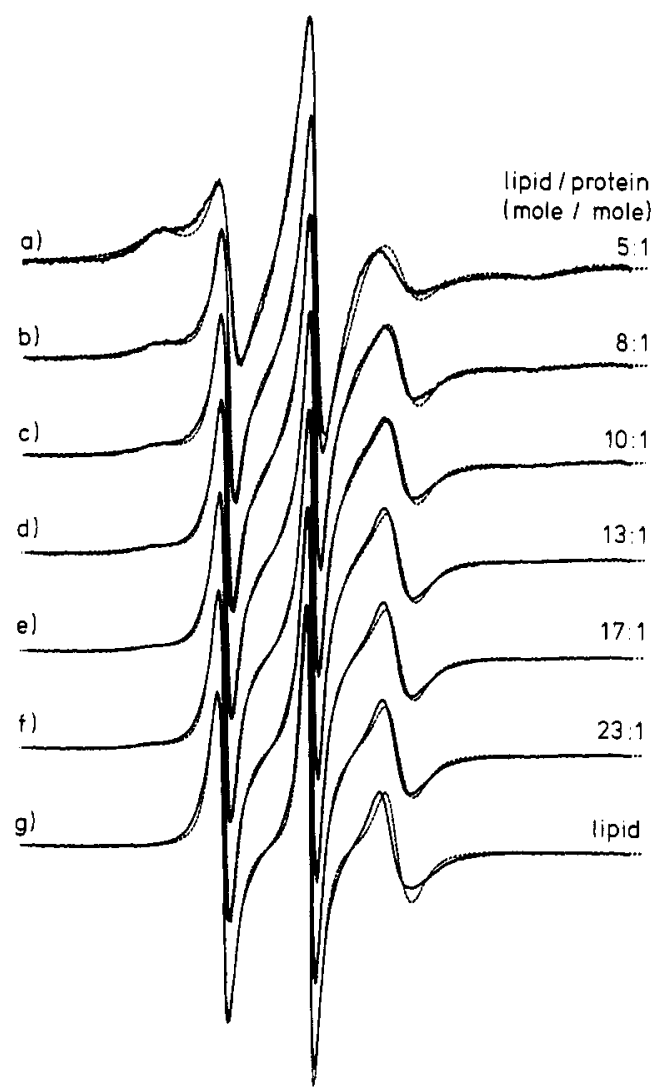

FIGURE 1: Experimental ESR spectra at $30^{\circ} \mathrm{C}$ (solid lines) and simulated spectra (dotted lines) of the 14-PCSL phosphatidylcholine spin-label in M13 coat protein/DMPC complexes as a function of lipid/protein mole ratio: (a) $5 / 1$, (b) $8 / 1$, (c) $10 / 1$, (d) $13 / 1$, (e) $17 / 1,(\mathrm{f}) 23 / 1,(\mathrm{~g})$ lipid alone. Total scan width: $10 \mathrm{mT}$. Simulation parameters are given in Table $I$.

this way are given in Figure 2 for the 14-PCSL, 14-PGSL, and 14-PASL labels as a function of the total $\mathrm{L} / \mathrm{P}$ ratio in the complexes. The experimental data have been analyzed according to the equation for the equilibrium lipid-protein association (Brotherus et al., 1981; Knowles et al., 1979):

$$
n_{\mathrm{f}}^{*} / n_{\mathrm{b}}{ }^{*}=\left(1 / K_{\mathrm{r}}\right)\left(n_{\mathrm{t}} / N_{\mathrm{l}}-1\right)
$$

where $n_{\mathrm{f}}{ }^{*} / n_{\mathrm{b}}{ }^{*}=(1-\mathrm{f}) / \mathrm{f}$ is the ratio of fluid to motionally restricted lipid component in the ESR spectrum, $n_{t}$ is the total $\mathrm{L} / \mathrm{P}$ ratio in the sample, and $N_{1}$ is the number of first-shell association sites on the protein. The approximation has been made in this equation that the concentration of the labeled lipid is small (experimentally less than $2 \%$ ) compared to that of the unlabeled lipid, and the average relative association constant, $K_{\mathrm{r}}$, is defined for the labeled lipid with respect to the unlabeled background host lipid (DMPC). The values of $K_{\mathrm{r}}$ and $N_{1}$, deduced from the linear regression lines in Figure 2, are, respectively, 2.1 and 4.4 for 14-PASL, 1.0 and 3.6 for 14-PGSL, and 1.0 and 4.0 for 14-PCSL. As can be seen, the number of first-shell association sites (ca. 4) is approximately the same for all three spin-labels used in this study. The relative association constant for 14-PCSL is close to unity, indicating that the spin-labeled phosphatidylcholine has the same affinity for the coat protein as the unlabeled bulk DMPC lipids. The 14-PGSL label has almost the same affinity as the 14-PCSL label. Spin-labeled phosphatidic acid, on the other hand, displays a marked selectivity for the protein, relative to phosphatidylcholine.

Simulations of the two-component spectra of the 14-PCSL label in the M13 coat protein/DMPC recombinants, performed according to the exchange-coupled Bloch equations,

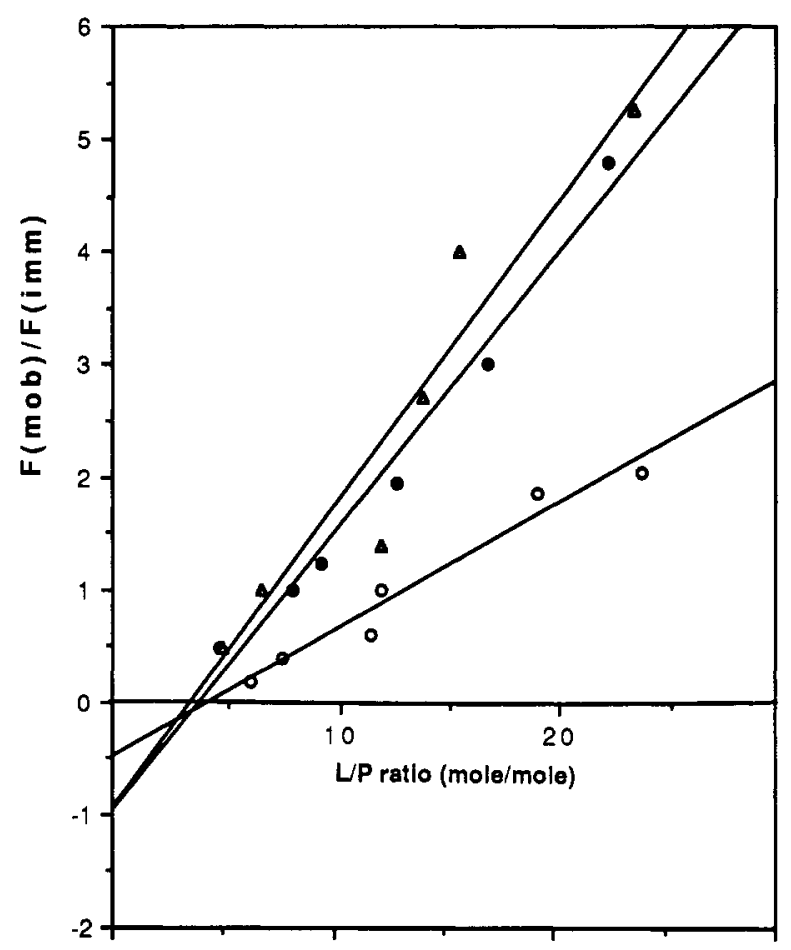

FIGURE 2: Lipid/protein titration of M13 coat protein/DMPC complexes from ESR difference spectra of the 14-PCSL $(\bullet)$, 14-PASL $(0)$, and 14-PGWL $(\Delta)$ lipid spin-labels at $30^{\circ} \mathrm{C}$. The ratio $F$ (mob) $/ F(\mathrm{imm})$ is the ratio of the double-integrated intensity of the fluid and motionally restricted components in the ESR spectra of the lipid-protein complexes, and $\mathrm{L} / \mathrm{P}$ is the total lipid/protein mole ratio.

Table I: Lipid/Protein Ratio (L/P) Dependence of the Fraction of Motionally Restricted Lipid, $f$, and the Exchange Off-Rate, $\tau_{b}{ }^{-1}$, Obtained from Simulations of ESR Spectra of the 14-PCSL, 14-PGSL, and 14-PASL Spin-Labels in M13 Coat Protein/DMPC Recombinants at $30^{\circ} \mathrm{C}$

\begin{tabular}{lccr}
\hline spin-label & L/P ratio (mol/mol) & $f$ & \multicolumn{1}{c}{$\tau_{\mathrm{b}}{ }^{-1}\left(\mathrm{~s}^{-1}\right)$} \\
\hline 14-PCSL & 4.8 & 0.78 & $5.7 \times 10^{6}$ \\
& 8.2 & 0.58 & $5.3 \times 10^{6}$ \\
& 9.5 & 0.51 & $4.7 \times 10^{6}$ \\
& 12.9 & 0.41 & $5.1 \times 10^{6}$ \\
& 17.0 & 0.31 & $5.7 \times 10^{6}$ \\
14-PGSL & 22.5 & 0.24 & $5.5 \times 10^{6}$ \\
& & & av $5.3 \times 10^{6}$ \\
& 5.0 & 0.80 & $4.5 \times 10^{6}$ \\
& 6.7 & 0.57 & $5.6 \times 10^{6}$ \\
& 12.2 & 0.49 & $6.0 \times 10^{6}$ \\
& 14.1 & 0.33 & $6.7 \times 10^{6}$ \\
14-PASL & 15.7 & 0.26 & $6.8 \times 10^{6}$ \\
& 23.6 & 0.23 & $6.9 \times 10^{6}$ \\
& & & av $6.1 \times 10^{6}$ \\
& 6.3 & 0.84 & $2.7 \times 10^{6}$ \\
& 7.9 & 0.78 & $2.4 \times 10^{6}$ \\
& 11.7 & 0.68 & $2.6 \times 10^{6}$ \\
& 12.2 & 0.55 & $2.5 \times 10^{6}$ \\
& 19.2 & 0.40 & $2.3 \times 10^{6}$ \\
& 24.0 & 0.37 & $2.1 \times 10^{6}$ \\
& & & av $2.4 \times 10^{6}$ \\
\hline
\end{tabular}

are given by the dotted lines in Figure 1. It is seen that a good correspondence is obtained between the simulated and experimental spectra over the entire range of $\mathrm{L} / \mathrm{P}$ ratios of the different recombinants. The exchange parameters used for the simulations are given in Table I, together with similar data for the 14-PGSL and 14-PASL spin-labels. As expected, the intrinsic off-rate, $\tau_{b}{ }^{-1}$, for exchange at the lipid-protein interface is independent of the $\mathrm{L} / \mathrm{P}$ ratio, except perhaps for 14-PGSL which shows a slight increase with increasing $L / P$ ratio. The values are given as an average. In contrast, the fraction of motionally restricted lipid decreases steadily with 

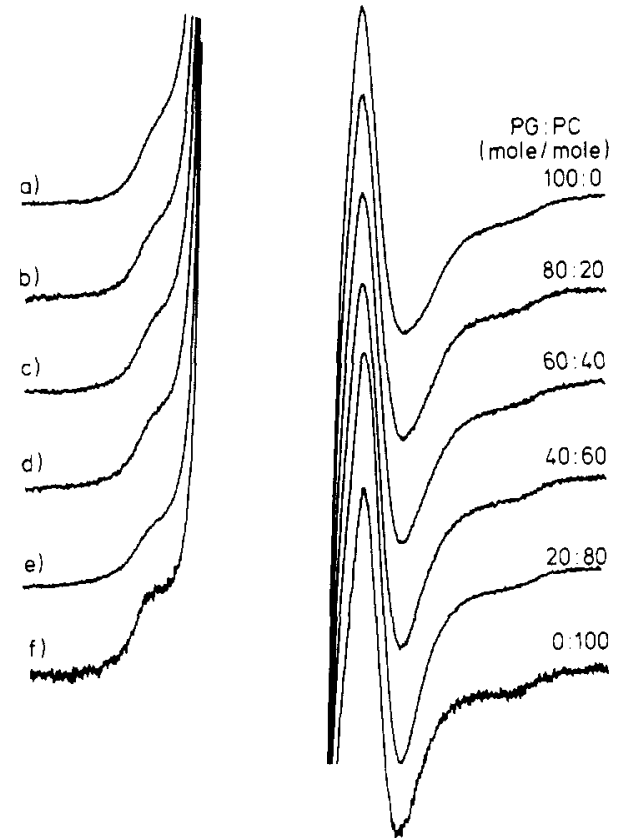

FIGURE 3: ESR spectra at $30^{\circ} \mathrm{C}$ of the 14-PCSL spin-label in M13 coat protein-DMPG/DMPC complexes of lipid/protein mole ratio $10 / 1$ and different DMPG/DMPC ratios: (a) DMPG alone; (b) $\mathrm{DMPG} / \mathrm{DMPC}=80 / 20 \mathrm{~mol} / \mathrm{mol} ;$ (c) $\mathrm{DMPG} / \mathrm{DMPC}=60 / 40$ $\mathrm{mol} / \mathrm{mol}$; (d) DMPG $/ \mathrm{DMPC}=40 / 60 \mathrm{~mol} / \mathrm{mol}$; (e) DMPG $/$ DMPC $=20 / 80 \mathrm{~mol} / \mathrm{mol}$; and (f) DMPC alone. The spectra are expanded vertically to show the increased resolution of the two components at higher DMPC contents in the complexes. Total scan width: $10 \mathrm{mT}$.

Table II: Dependence of the Fraction of Motionally Restricted Lipid, $f$, and the Exchange On- and Off-Rate, $\tau_{\mathrm{f}}^{-1}$ and $\tau_{\mathrm{b}}^{-1}$, on DMPC/DMPG Mole Ratio ${ }^{\circ}$

\begin{tabular}{|c|c|c|c|c|c|c|}
\hline \multirow[b]{2}{*}{$\begin{array}{c}\text { DMPC/ } \\
\text { DMPG } \\
(\mathrm{mol} / \mathrm{mol})\end{array}$} & \multicolumn{3}{|c|}{ 14-PGSL } & \multicolumn{3}{|c|}{ 14-PCSL } \\
\hline & $f$ & $\begin{array}{c}x_{f}^{-1} \\
\times 10^{-6} \\
\left(s^{-1}\right)\end{array}$ & $\begin{array}{c}\tau_{\mathrm{b}}{ }^{-1} \\
\times 10^{-6} \\
\left(\mathrm{~s}^{-1}\right)\end{array}$ & $f$ & $\begin{array}{c}\tau_{f}^{-1} \\
\times 10^{-6} \\
\left(\mathrm{~s}^{-1}\right)\end{array}$ & $\begin{array}{c}\tau_{b^{-1}} \\
\times 10^{-6} \\
\left(\mathrm{~s}^{-1}\right)\end{array}$ \\
\hline $100 / 0$ & 0.56 & 7.2 & 5.7 & 0.58 & 6.5 & 4.7 \\
\hline $80 / 20$ & 0.60 & 9.7 & 6.5 & 0.55 & 7.5 & 9.2 \\
\hline $60 / 40$ & 0.60 & 13.6 & 9.1 & 0.52 & 11.5 & 10.6 \\
\hline $40 / 60$ & 0.61 & 16.0 & 10.2 & 0.53 & 13.4 & 11.9 \\
\hline $20 / 80$ & 0.62 & 22.5 & 13.8 & 0.52 & 15.9 & 14.7 \\
\hline $0 / 100$ & 0.58 & 18.8 & 16.7 & 0.54 & 19.1 & 16.2 \\
\hline
\end{tabular}

a Obtained from simulations of the ESR spectra of the 14-PCSL and 14-PGSL spin-label in M13 coat protein/mixed-lipid recombinants at $30^{\circ} \mathrm{C}$.

increasing $\mathrm{L} / \mathrm{P}$ ratio, as shown in Figure 2.

To investigate further the interactions with negatively charged lipids, the M13 coat protein was also reconstituted in mixed bilayers of DMPC and DMPG at various mole fractions of the two lipids.

There are significant and progressive changes in the spectral line shapes as a function of DMPG content in the mixed-lipid systems. This is illustrated by the vertically expanded displays of the ESR spectra of 14-PCSL in Figure 3. As the mole fraction of DMPG is increased, the line width of both spectral components increases, as is indicated by the increasing overlap of the two spectral components in Figure 3. The changes in line shape make it difficult to match the fluid component exactly in spectral subtractions. Therefore, in this case, the quantitation relies heavily on simulations of the exchangecoupled two-component spectra. The simulation parameters are given in Table II. The on-rates, $\tau_{f}^{-1}$, given in Table II are not intrinsic on-rates but are simply related to the intrinsic off-rates, $\tau_{b}^{-1}$, and the fraction of the label in the motionally

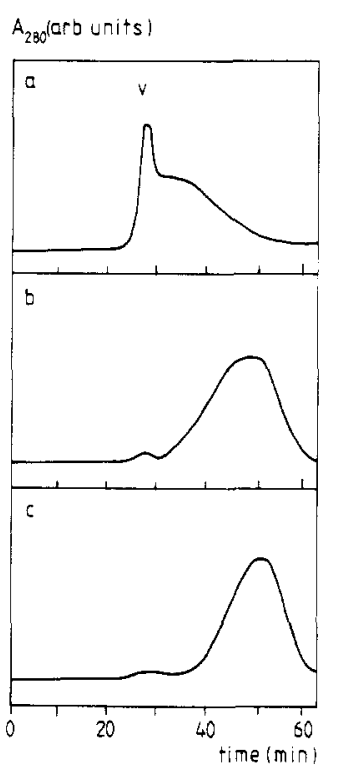

FIGURE 4: Superose-6 high-performance gel filtration chromatograms of M13 coat protein aggregates after solubilization in cholate. $V$ indicates the void volume of the column. The lipid composition of the original complexes was (a) 100\% DMPC, (b) $80 / 20 \mathrm{~mol} / \mathrm{mol}$ DMPC/DMPG, and (c) 100\% DMPG, and the lipid/protein ratio $=20 / 1$.

Table III: Retention Time of the Protein Aggregates on Superose-6 Gel Filtration of Samples with Different DMPC/DMPG Lipid Ratios $^{a}$

\begin{tabular}{ccc}
\hline $\begin{array}{c}\text { DMPC/DMPG } \\
(\mathrm{mol} / \mathrm{mol})\end{array}$ & $\begin{array}{c}\text { retention } \\
\text { time (min) }\end{array}$ & aggregation no. \\
\hline $100 / 0$ & 37.5 & $400 \pm 40$ \\
$80 / 20$ & 50.0 & $60 \pm 6$ \\
$60 / 40$ & 52.5 & $40 \pm 4$ \\
$40 / 60$ & 54.0 & $30 \pm 3$ \\
$20 / 80$ & 55.0 & $30 \pm 3$ \\
$0 / 100$ & 54.0 & $30 \pm 3$ \\
\hline
\end{tabular}

${ }^{a}$ Determined according to Spruijt et al. (1989).

restricted component by the equation for material balance (Marsh, 1985):

$$
\tau_{\mathrm{b}}^{-1} f=\tau_{\mathrm{f}}^{-1}(1-f)
$$

The 14-PGSL label shows a selectivity relative to 14-PCSL in mixtures with around equimolar proportions of DMPC and DMPG, but none in DMPC or DMPG alone.

The tryptophan fluorescence of M13 coat protein as a function of the DMPG content of the bilayer showed an increase in fluorescence intensity from 0 to $40 \%(w / w)$ DMPG content of the lipid system. This increase in fluorescence gives an indication of a change in the number of protein-protein contacts, so probably smaller aggregates are present. Further addition of DMPG to the lipid system [40-100\% (w/w)] only slightly increases the fluorescence intensity any further. Information about the state of aggregation of M13 coat protein was obtained from gel filtration chromatography, performed after solubilization of the lipid-protein recombinants in cholate. The result is given in Figure 4. In pure DMPC (Figure 4a), a sharp peak is seen at 30 min superimposed on a broad peak with a maximum at about $40 \mathrm{~min}$. With $20 \%(\mathrm{w} / \mathrm{w})$ DMPG in the lipid system, the peak at $30 \mathrm{~min}$ is decreased, and the broad peak is shifted from 40 to 50 min (Figure $4 b$ ). The 50-min peak becomes smaller for protein complexes with pure DMPG (Figure 4c). After calibration of the column with marker proteins, molecular weights were determined according to Spruijt et al. (1989), and the aggregation numbers in the 
cholate extracts are given in Table III. This analysis gives an estimate of the minimum aggregation number in the original lipid-protein system. After cross-linking the protein with glutaraldehyde (while still in the vesicle), all proteins elutes after $30 \mathrm{~min}$ from the column, indicating the formation of giant protein complexes (data not shown).

\section{Discussion}

Previous studies of lipid-protein interactions using spin-label ESR spectroscopy have concentrated mainly either on reconstituted systems in a single lipid background (mostly phosphatidylcholine) or on membranes containig a natural lipid mixture. For instance, cytochrome oxidase has been reconstituted with various well-defined phosphatidylcholine species (Knowles et al., 1981; Griffith et al., 1986). Both the acetylcholine receptor (Marsh \& Barrantes, 1978; Ellena et al., 1983) and rhodopsin (Watts et al., 1979; Ryba et al., 1987) have been investigated in natural membranes and reconstituted with defined phosphatidylcholines. Similarly the $\mathrm{Na}^{+}, \mathrm{K}^{+}$. ATPase has also been studied in natural membranes and reconstituted with phosphatidylcholine (Esmann et al., 1985; Brotherus et al., 1981). Myelin proteolipid protein has also been studied in dimyristoylphosphatidylcholine recombinants (Brophy et al., 1984). In one case, myelin basic protein has been studied in its interaction with mixed phosphatidylcholine/phosphatidylglycerol bilayers, and some evidence for specific molecular associations between the negatively charged lipid and protein has been found by ${ }^{2} \mathrm{H}$ NMR (Sixl et al., 1984; Watts, 1987).

This work is one of the few studies of lipid-protein interactions in which reconstitution has been made with negatively charged lipid alone, and in which the ratio of negatively charged to zwitterionic lipid has been varied systematically. This represents a controlled approach to the complexity of the lipid composition of natural membranes.

The L/P titrations reveal that an approximately constant number of nonselective lipids (phosphatidylcholine and phosphatidylglycerol) are associated with the M13 coat protein in DMPC complexes, independent of the total $\mathrm{L} / \mathrm{P}$ ratio. The low number of these association sites (ca. 4 per protein monomer) is consistent with the protein being present in the membrane in an oligomeric state in which some potential association sites are shielded. Protein aggregation for the reconstituted M13 coat protein has been detected by using other techniques such as time-resolved tryptophan fluorescence (Datema et al., 1987b) and ${ }^{2}$ H NMR (Datema et al., 1988). A decreased number of binding sites caused by protein aggregation has been suggested also for myelin proteolipid protein (Brophy et al., 1984). For the M13 coat protein monomer, it can be calculated that a single membrane-spanning $\alpha$-helix could accommodate approximately 10 phospholipids around its perimeter. To reduce this value to 4 would require that the protein be minimally a tetramer and that the lipids be totally excluded from the regions of protein-protein contact. However, the aggregation number reported in Table III, which is 100 times larger, suggests that the situation is more complicated than this, as will be discussed below.

Further, we see a different selectivity pattern for spin-labeled lipids in the pure DMPC lipid system as compared to our previous study in the mixed-lipid system (Datema et al., 1987a). The selectivity of the M13 coat protein for the 14PCSL spin-label becomes the same as for the 14-PGSL spin-label. This is reflected by the essentially identical values of $K_{\mathrm{r}}$ as presented above. One reason for this difference in selectivity might arise from different properties of the background lipid with which the spin-labels are competing. The association constant $K_{\mathrm{r}}$, is defined relative to the spin-label at high dilution in the host lipid as standard states (Brotherus et al., 1981). Thus, differences in selectivity can arise both from changes in the nature of the lipid-protein interface and from changes in the properties of the bulk lipid. Changes in the former could arise either from differences in the conformation of the protein, which is not seen by circular dichroism (data not shown), or from differences in the nature of the lipid-protein aggregates (see below). A distinctly higher value of $K_{\mathrm{r}}$ is found for the 14-PASL spin-labeled phosphatidic acid. This is greater by a factor of 2 for phosphatidic acid than for 14-PGSL phosphatidylglycerol and is of the same order of magnitude as found previously in the mixed-lipid system (Datema et al., 1987a). The specificity of interaction with certain of the negatively charged lipids, found both in this and in the previous work, is presumably, at least in part, related to the concentration of positively charged residues in the carboxy-terminal part of the protein. The difference in selectivity of the coat protein is also seen in the intrinsic exchange off-rates of the lipids $\left(\tau_{b}{ }^{-1}\right)$, as summarized in Table I. An increase in selectivity results in a decrease in the off-rate. Quantitatively, this relation between the thermodynamics of the lipid selectivity and the exchange dynamics can be seen by combining eq 1 and 2 . Assuming that the on-rates are the same for the different lipids (i.e., diffusion-controlled), then the ratio of the intrinsic off-rate constants for two lipids, e.g., $\mathrm{L}_{1}$ and $\mathrm{L}_{2}$, is given by (Marsh, 1985; Horvăth et al., 1988)

$$
\tau_{\mathrm{b}}{ }^{-1}\left(\mathrm{~L}_{2}\right) / \tau_{\mathrm{b}}{ }^{-1}\left(\mathrm{~L}_{1}\right)=K_{\mathrm{r}}\left(\mathrm{L}_{1}\right) / K_{\mathrm{r}}\left(\mathrm{L}_{2}\right)
$$

For the 14-PCSL, 14-PGSL, and 14-PASL spin-labeled phospholipids, the ratios of the relative association constants are 1.0:1.0:2.0, compared with the ratios of the inverse rate constants of 1.0:0.9:2.2, respectively. This is in good agreement with the predictions of eq 3 and further substantiates the methods used for analyzing the selectivity and exchange dynamics exhibited by the two-component lipid spectra.

The absolute values of the lipid exchange rate constants in the DMPC system are also of interest. The value for 14PCSL, which exhibits no relative selectivity for the protein, is $5 \times 10^{6} \mathrm{~s}^{-1}$ at $30^{\circ} \mathrm{C}$. This value is significantly smaller than the off-rate found with the myelin proteolipid protein (Horvath et al., 1988), rhodopsin (Ryba et al., 1987), and the $\mathrm{Ca}^{2+}$. ATPase (East et al., 1985). The off-rate is also considerably slower than the rate of free lipid diffusion in DMPC bilayers. Values for the latter have been determined to be $\tau_{\text {diff }}{ }^{-1}=3.7$ $\times 10^{7} \mathrm{~s}^{-1}$ by photobleaching (Vaz et al., 1985) and $7.5 \times 10^{7}$ $\mathrm{s}^{-1}$ for spin-labeled phosphatidylcholine by ESR spectroscopy (Sachse et al., 1987) in DMPC bilayers at $30^{\circ} \mathrm{C}$. The difference between the intrinsic off-rate and the average free diffusion rate can be expressed in terms of an effective association constant $K_{\text {eff }}(\mathrm{av})=\tau_{\text {diff }}{ }^{-1} / \tau_{\mathrm{b}}{ }^{-1}=7-14$, compared with values of $K_{\text {eff }}(\mathrm{av})=2-5$ obtained for the myelin proteolipid protein (Horvăth et al., 1988) and rhodopsin (Ryba et al., 1987). This intrinsically stronger association and the difference in off-rates already noted point to a significant difference in the nature of the lipid-protein interface for these systems.

So far, we have assumed that all the lipids participate in the exchange process. However, recent ${ }^{2} \mathrm{H}$ NMR experiments of Van Gorkom et al. (1989) suggest that part of the lipids in the same lipid-protein system are trapped and have an exchange rate smaller than $10^{3} \mathrm{~s}^{-1}$, i.e., would not participate in exchange in our experiments. From the experimental exchange rates, then, the fraction of lipids which do participate in exchange could be estimated if it is assumed that the actual off-rate of the nontrapped lipids is given by $1.6 \times 10^{7} \mathrm{~s}^{-1}$, as found for all other proteins investigated so far in DMPC 
(Horvath et al., 1988; Ryba et al., 1987; East et al., 1985). Since the on-rate is diffusion-controlled and therefore is constant, it can be shown from eq 2 that

$$
\begin{aligned}
& {[(1-f) / f]_{\text {actual }} /[(1-f) / f]_{\text {experimental }}=} \\
& \qquad\left(\tau_{\mathrm{b}}^{-1}\right)_{\text {actual }} /\left(\tau_{\mathrm{b}}{ }^{-1}\right)_{\text {experimental }}
\end{aligned}
$$

From eq 1 , it can further be shown that this ratio is equal to the fraction of protein-associated lipid that is exchanging. When data from Table I are used, this would predict that one-third of the 14-PCSL labels associated with the protein are available for exchange in the DMPC system. This is in, at least qualitative, agreement with the estimate of Van Gorkom et al. (1989).

From Figure 3, it is clear that the widths of the ESR lines increase when DMPG is introduced into the lipid system. This results in an increasing exchange rate, found by simulation, as given in Table II, neglecting the presence of trapped lipids. The exchange rate found for the DMPG lipid system resembles the exchange rate found for the other DMPC/protein systems (see above), i.e., $1.6 \times 10^{7} \mathrm{~s}^{-1}$. This indicates the absence of trapped lipid in the DMPG/protein system. Therefore, the observed increase in exchange rate on going from pure DMPC to the pure DMPG lipid-protein system reflects a decrease of the amount of trapped lipids. This decrease in the amount of trapped lipids could be a result of an increase in space between the protein aggregates as is suggested by the fluorescence results. A larger separation between protein aggregates would decrease the fluorescence quenching of the tryptophan residue with increasing DMPG content of the lipid-protein complexes, as is seen. In pure DMPC, the protein aggregates are therefore thought to be tightly packed with trapped lipids enclosed. Adding DMPG to the lipid system increases the space available for the lipids within the protein complex, thereby decreasing the amount of trapped lipids. At contents of $40 \%(w / w)$ DMPG or higher in the system, the influence of DMPG relatively diminishes, as deduced from the fluorescence quenching experiments. This suggests that the distance between protein aggregates is then large enough to cause no additional quenching. The amount of trapped lipids, as reflected by the increased exchange rate, however, continues to decrease up to DMPG contents of $100 \%$ in the lipid-protein system, as seen in Table II. This indicates that the trapped lipids do not normally exchange with the bulk lipids. Only when the space between the proteins is increased even more (hardly detectable by fluorescence increase anymore) do these trapped lipids exchange normally.

Adding DMPG to the lipid system not only changes the packing of the protein aggregates within a larger protein complex but also decreases the overall size of the aggregates. The largest effect occurs from 0-20\% (w/w) DMPG present in the vesicle (see Table III), Considering the amount of phosphatidylglycerol present in the $E$. coli host membrane, $19 \%(w / w)$ (Burnell et al., 1980), the stabilizing contribution of phosphatidylglycerol for smaller coat protein aggregates might play a role during the membrane-associated M13 bacteriophage assembly and disassembly.

\section{ACKNOWLEDGMENTS}

We thank Ruud B. Spruijt for biochemical assistance, Leon van Gorkom for preparing the phospholipid spin-labels, and Peter Fisher for preparing 14-stearic acid spin-labels.

Registry No. DMPC, 18194-24-6; DMPG, 61361-72-6.

\section{REFERENCES}

Armstrong, J., Hewitt, J. A., \& Perham, R. N. (1983) EMBO J. 2, 1641-1646.
Bartlett, G. R. (1959) J. Biol. Chem. 234, 466-468.

Beck E., Sommer, R., Auerswald, E., Kurz, C., Zink, B., Oosterburg, G., Schaller, M., Sugimoto, K., Sugisaki, H., Okamoto, T. \& Takanami, M. (1978) Nucleic Acids Res. 5, 4495-4503.

Brophy, P. J., Horväth, L. I., \& Marsh, D. (1984) Biochemistry 23, 860-865.

Brotherus, J. R., Griffith, O. H., Brotherus, M. A., Jost, P. C., Silvius, J. R., \& Hokin, L. E. (1981) Biochemistry 20, 5261-5267.

Burnell, E., van Alphen, L., Verkleij, A., \& de Kruijff, B. (1980) Biochim. Biophys. Acta 597, 492-501.

Chamberlain, B., Nozaki, Y., Tanford, C., \& Webster, R. (1978) Biochim. Biophys. Acta 510, 18-37.

Datema, K. P., Wolfs, C. J. A. M., Marsh, D., Watts, A., \& Hemminga, M. A. (1987a) Biochemistry 26, 7571-7574.

Datema, K. P., Visser, A. J. W. G., van Hoek, A., Wolfs, C. J. A. M., Spruijt, R. B., \& Hemminga, M. A. (1987b) Biochemistry 26, 6145-6152.

Datema, K. P., van Boxtel, B. J. H., \& Hemminga, M. A. (1988) J. Magn. Reson. 77, 372-376.

Davoust, J., \& Devaux, P. F. (1982) J. Magn. Reson. 48, 475-494.

East, J. M., Melville, D., \& Lee, A. G. (1985) Biochemistry 24, 2615-2623.

Ellena, J. F., Blazing, M. A., \& McNamee, M. G. (1983) Biochemistry 22, 5523-5535.

Esmann, M., Watts, A., \& Marsh, D. (1985) Biochemistry 24, 3572-3578.

Garssen, G. J., Hilbers, C. W., Schoenmaker, J. G. G., \& Van Boom, J. H. (1977) Eur. J. Biochem. 81, 853-863.

Griffith, O. H., McMillen, D. A., Keana, J. F. W., \& Jost, P. C. (1986) Biochemistry 25, 574-584.

Hagen, S., Weiner, J., \& Sykes, B. D. (1978) Biochemistry 17, 3860-3866.

Horvåth, L. I., Brophy, P. J., \& Marsh, D. (1988) Biochemistry 27, 46-52.

Knippers, R., \& Hoffmann-Berling, H. (1966) J. Mol. Biol. 21, 281-292.

Knowles, P. F., Watts, A. \& Marsh, D. (1979) Biochemistry $18,4480-4487$.

Knowles, P. F., Watts, A., \& Marsh, D. (1981) Biochemistry 20, 5888-5894.

Kühn, A., Wickner, W., \& Kreil, G. (1986) Nature 322, 335-339.

Marsh. D. (1982) Tech. Life Sci.: Biochem. B4/II, B426/ $1-B 426 / 44$.

Marsh D. (1985) in Progress in Protein-Lipid Interactions (Watts, A., \& De Pont, J. J. H. H. M., Eds.) Chapter 4, pp 143-172, Elsevier, Amsterdam.

Marsh, D., \& Barrantes, F. J. (1978) Proc. Natl. Acad. Sci. U.S.A. 75, 4329-4333.

Marsh, D., \& Watts, A. (1982) in Lipid-Protein Interactions (Jost, P. C., \& Griffith, O. H., Eds.) Vol. II, pp 53-126, Wiley-Interscience, New York.

Marvin, D. A., \& Wachtel, E. J. (1975) Nature 253, 19-23.

Peterson, G. L. (1977) Anal. Biochem. 83, 346-356.

Ray, D. S. (1977) in Comprehensive Virology (FraenckelConrad, H., \& Wagner, R. R., Eds.) Vol. 7, pp 105-178, Plenum Press, New York.

Ryba, N. J. P., Horvăth, L. I., Watts, A. \& Marsh, D. (1987) Biochemistry 26, 3234-3240.

Sachse, J.-H., King, M. D., \& Marsh, D. (1987) J. Magn. Reson. 71, 385-404. 
Sixl, F., Brophy, P. J., \& Watts, A. (1984) Biochemistry 23, 2032-2039.

Spruijt, R. B., Wolfs, C. J. A. M., \& Hemminga, M. A. (1989) Biochemistry 28, 9158-9165.

Van Gorkom, L. C. M., Horvăth, L. I., Sternberg, B., Hemminga, M. A., \& Watts, A. (1989) Biochemistry (submitted for publication).
Van Wezenbeek, P. M. G. F., Hulsebos, T. J. M., \& Schoenmakers, J. G. G. (1980) Gene 11, 129-148.

Vaz, W. L. C., Clegg, R. M., \& Hallmann, D. (1985) Biochemistry 24, 781-786.

Watts, A. (1987) J. Bioenerg. Biomembr. 19, 625-653.

Watts, A., Volotovski, I. D., \& Marsh, D. (1979) Biochemistry 18, 5006-5013.

\title{
NMR Distance Measurements in DNA Duplexes: Sugars and Bases Have the Same Correlation Times ${ }^{\dagger}$
}

\author{
Brian R. Reid, ${ }^{*, 1,8}$ Kevin Banks, ${ }^{\ddagger}$ Peter Flynn, ${ }^{\ddagger}$ and Willy Nerdal ${ }^{\ddagger}, \|$ \\ Departments of Chemistry and Biochemistry, University of Washington, Seattle, Washington 98195 \\ Received August 1, 1988; Revised Manuscript Received July 28, 1989
}

\begin{abstract}
To evaluate whether the sugar moieties of short DNA duplexes exhibit local motion of sufficient amplitude to affect interproton distance measurements, we have carried out a series of time-dependent NOESY experiments at increasingly shorter mixing times on dodecamer DNA duplexes. By use of the cytosine $\mathrm{H} 5-\mathrm{H} 6$ vector as a known distance in the bases and the geminal $2^{\prime} \mathrm{H}-2^{\prime \prime} \mathrm{H}$ vector as a known distance in the sugars, the corresponding apparent cross-relaxation rates were sampled at various mixing times. While the ratio of the inverse sixth power of these two fixed distances is in the range 6-7, when the system is sampled at $100 \mathrm{~ms}$ the apparent initial rate of growth of the $2^{\prime} \mathrm{H}-2^{\prime \prime} \mathrm{H}$ NOESY crosspeak is only 1.9-2.0 times faster than that of the H5-H6 crosspeak-in agreement with the results of Clore and Gronenborn [Clore, G. M., \& Gronenborn, A. M. (1984) FEBS Lett. 172, 219; (1984) FEBS Lett. 175, 117] and of Gronenborn and Clore [Gronenborn, A. M., \& Clore, G. M. (1985) Prog. NMR Spectrosc. 17, 1]. This observation was interpreted to indicate the existence of internal mobility with a 3 -fold shorter correlation time for the sugar moieties in DNA and led to the use of this shorter correlation time to estimate sugar-sugar proton distances and many sugar-base proton distances in subsequent DNA structure determination. We have examined $2^{\prime} \mathrm{H}-2^{\prime \prime} \mathrm{H}$ cross-relaxation and $\mathrm{H} 5-\mathrm{H} 6$ cross-relaxation at $100,90,60,30$, and $15 \mathrm{~ms}$ in dodecamer DNA duplexes. The ratio of the rates increases smoothly from $1.9-2.0$ to $6-7$ as the mixing time is reduced. The results show that the scaling discrepancy at $100 \mathrm{~ms}$ is not due to internal motion in the sugar but is simply due to spin diffusion from the rapidly relaxing $2^{\prime} \mathrm{H}, 2^{\prime \prime} \mathrm{H}$ geminal protons. The lack of significant largeamplitude nanosecond local motion in the sugars casts doubt on the accuracy of interproton distances measured on the assumption of a 3-fold shorter correlation time for sugar protons and hence on the validity of DNA structures generated from such distances.
\end{abstract}

The he development of high-field NMR ${ }^{1}$ spectrometers operating in the $500-\mathrm{MHz}$ range has led to the ability to determine the structures of small proteins (Havel \& Wüthrich, 1985; Williamson et al., 1986) and short DNA duplexes (Hare \& Reid, 1986; Nilsson et al., 1986; Hare et al., 1986a,b; Nilges et al., 1987a,b) directly in solution. Common to all these studies is the measurement of distances between assigned protons of known residues within the polymer [for reviews, see Wemmer and Reid (1985), Gronenborn and Clore (1985), and Reid (1987)]. In the case of DNA structure determination, these interproton distances can be used as constraints, either to refine an idealized model double helix (Nilsson et al., 1986; Nilges et al., 1987a,b; Clore et al., 1985) or to calculate a structure de novo by using distance geometry methods (Hare \& Reid, 1986; Hare et al., 1986a,b).

The interproton distances to be used in calculating a DNA structure or in refining a model duplex by restrained least squares (Clore et al., 1985) or by restrained molecular dy-

\footnotetext{
† Supported by NIH Grant PO1 GM32681.

$\ddagger$ Department of Chemistry.

Department of Biochemistry.

IOn leave from the Chemistry Department, University of Bergen, Norway.
}

namics (Nilsson et al., 1986; Nilges et al., 1987a,b) are experimentally obtained from time-dependent NOE measurements using either one-dimensional (Nilsson et al., 1986; Nilges et al., 1987a) or two-dimensional (Hare \& Reid, 1986; Nilges et al., 1987b) NMR data. The determination of interproton distances from NOE buildup rates involves both approximations and assumptions, either of which can jeopardize the accuracy of the distance measurement and hence the correctness of the resulting structure.

The approximation used in NOE distance measurement is the two-spin approximation in which only the rate of dipolar magnetization transfer between proximal spins $i$ and $j$ is monitored, and all other spins are ignored. For a two-spin system, the rate of magnetization transfer is (Solomon, 1955)

$$
\begin{aligned}
R_{1(i j)}=\frac{\gamma^{4} \hbar^{2}}{10 r_{i j}^{6}}\left[\frac{6 \tau_{\mathrm{c}}}{1+4 \omega^{2} \tau_{\mathrm{c}}{ }^{2}}\right. & \left.-\tau_{\mathrm{c}}\right]= \\
& -\frac{\gamma^{4} \hbar^{2} \tau_{\mathrm{c}}}{10 r_{i j}{ }^{6}} \quad \text { when } \omega \tau_{\mathrm{c}} \gg 1
\end{aligned}
$$

\footnotetext{
1 Abbreviations: NOE, nuclear Overhauser effect; NOESY, nuclear Overhauser effect spectroscopy; NMR, nuclear magnetic resonance; DNA, deoxyribonucleic acid.
} 